[0212-7199 (2007) 24: 4; pp 187-189] ANALES DE MEDICINA INTERNA Copyright (C) 2007 ARAN EDICIONES, S.L.

AN. MED. INTERNA (Madrid) Vol. 24, N. ${ }^{\circ} 4$, pp. 187-189, 2007

\title{
Neumonía lipoidea en relación con una aspiración accidental de gasóleo
}

\author{
M. A. AIBAR ARREGUI, K. LABORDA EZQUERRA, F. CONGET LÓPEZ \\ Servicio de Neumología. Hospital Clínico Universitario Lozano Blesa. Zaragoza
}

LIPOID PNEUMONIA RELATED TO AN ACCIDENTAL ASPIRATION

OF GAS-OIL

\begin{abstract}
RESUMEN
Se presenta el caso de un varón de 35 años, sin historia previa de patología pulmonar crónica y no fumador, que acude al servicio de urgencias tras la aspiración accidental de gasóleo y desarrolla en el curso de unas horas una neumonía lipoidea. En la tomografía computadorizada (TC) se observa una zona en vidrio deslustrado en lóbulo medio que, junto a la presencia del antecedente epidemiológico inmediato, permite establecer el diagnóstico. Tras un año de seguimiento el paciente permanece estable y asintomático, aunque persisten en la TC leves secuelas del proceso agudo.
\end{abstract}

PALABRAS CLAVE: Neumonía lipoidea. Aspiración. Gasóleo.

\begin{abstract}
A 35-year-old non smoker man with no known history of chronic pulmonary disease, was treated at our hospital after accidental aspiration of gas-oil. He had developed an acute lipoid pneumonia in a few hours. Computed tomography of the chest showed a ground-glass pattern in middle lobe; given the immediate epidemiological precedent, it was possible to confirm a definitive diagnosis. One year later the patient is asymptomatic although small signs of acute process remain in the computed tomography.
\end{abstract}

KEY WORDS: Lipoid pneumonia. Aspiration. Gas-oil. Aibar arregui MA, Laborda Ezquerra K, Conget López F. Neumonía lipoidea en relación con una aspiración accidental de gasóleo. An Med
Interna (Madrid) 2007; 24: 187-189.

\section{INTRODUCCIÓN}

La neumonía lipoidea exógena es una entidad poco frecuente, y a menudo infradiagnosticada, que se produce como consecuencia de la inhalación de materiales grasos. Dicha inhalación puede ser crónica e incluso imperceptible por el propio afectado o, menos frecuentemente, consecuencia de una exposición única de mayor o menor intensidad que condiciona la aparición de una neumonía aguda.

En los casos crónicos, la clínica puede ser inespecífica e incluso inexistente durante periodos variables de tiempo, en los que tanto la función respiratoria como el patrón radiológico son también variables y a menudo inespecíficos. En la mayoría de estos casos, el diagnóstico definitivo exige la realización de una biopsia pulmonar para el estudio histológico.

Los casos de neumonía lipoidea aguda, si bien menos frecuentes, son relativamente más sencillos de diagnosticar debido al antecedente epidemiológico inmediato, aunque para ello es precisa, obviamente, la recogida correcta de dicho antecedente.

En este artículo se presenta el caso de un paciente con una aspiración accidental de gasóleo que desarrollo una neumonía lipoidea aguda bilateral a las pocas horas de la exposición.

\section{CASO APORTADO}

Varón de 35 años, no fumador y sin antecedentes patológicos de interés, salvo una TBC sufrida tres años atrás, y alergia a rifampicina. Trabajaba en una gasolinera y sufrió una cuantiosa aspiración accidental de gasóleo. El paciente acudió al servicio de urgencias de nuestro hospital presentando únicamente tos no productiva y sin referir pirosis ni disfagia; la exploración física fue normal salvo por el intenso enrojecimiento de la faringe, la auscultación cardiaca y la pulmonar fueron normales en el momento de su admisión en el servicio de urgencias.

Trabajo aceptado: 227 de noviembre de 2006 
Tanto el ECG como la radiografía de tórax realizados en ese momento fueron también normales. En la analítica destacaba una moderada leucocitosis $\left(17.400 / \mathrm{mm}^{3}\right)$ con marcada neutrofilia $(92,9 \%)$, y en la gasometría arterial basal una alcalosis respiratoria con los siguientes parámetros: $\mathrm{pH} 7,52 \mathrm{pO}_{2} 90 \mathrm{mmHg}, \mathrm{pCO}_{2} 24,5$ $\mathrm{mmHg}$, Saturación $98,1 \% \mathrm{CO} 3 \mathrm{H} 20,6 \mathrm{mmol} / \mathrm{l}$ y una tasa de carboxihemoglobina del 1,2\%.

El paciente permanece en observación con fluidoterapia y corticoides intravenosos, presentando a las pocas horas del ingreso dolor pleurítico acompañado de un pico febril de $39,5^{\circ} \mathrm{C}$ por lo que se practica una nueva radiografía de tórax que evidencia la presencia de una consolidación parcheada en lóbulo medio y más leve en ambas bases pulmonares. A la vista de estos datos el paciente ingresa en el servicio de neumología añadiéndose al tratamiento un antibiótico de amplio espectro, gastroprotectores y un AINE.

Se realizó una TAC con contraste que confirmó la consolidación en segmento 6 de lóbulo medio y área en vidrio deslustrado compatible con neumonía lipoidea, descartándose otros diagnósti$\cos$ debido al antecedente epidemiológico inmediato de este paciente (Fig. 1).

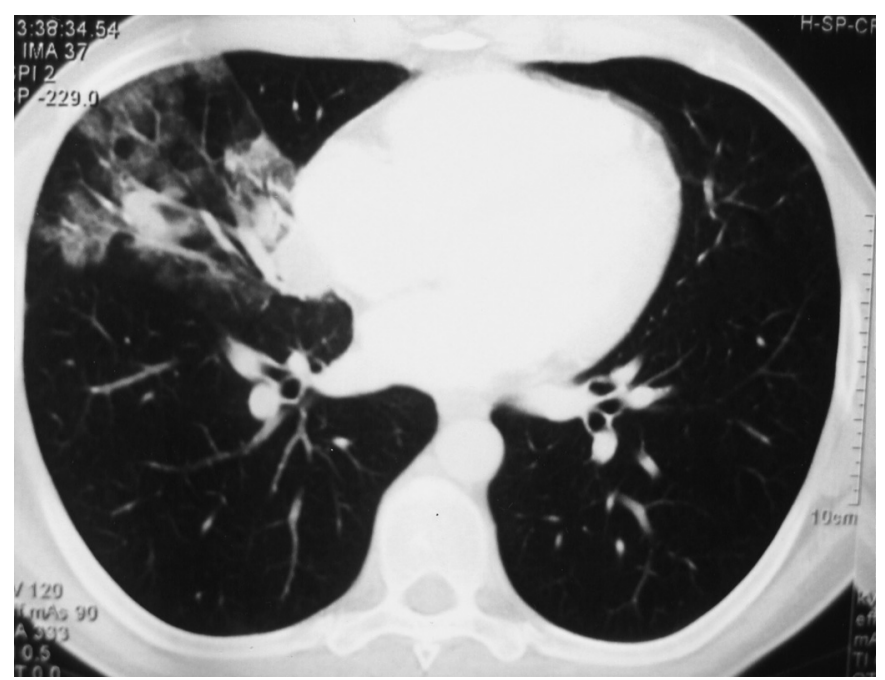

Fig. 1. TAC durante el proceso agudo.

En la fibrobroncoscopia aparecieron signos inflamatorios y en el broncoaspirado se descartó la presencia de células tumorales. Durante la misma maniobra se realizó un lavado broncoalveolar (LBA) para estudio citológico en el que se informó la presencia de un $98 \%$ de macrófagos pero no se pudo constatar la presencia de vacuolas grasas en su interior; el estudio del líquido mediante cromatografía de gases por el laboratorio de toxicología no pudo demostrar la presencia de hidrocarburos.

La evolución del paciente fue buena con el tratamiento instaurado, persistiendo durante 5 días tanto los picos febriles como la tos; el paciente refería un intenso olor y sabor a gasóleo coincidiendo con alguno de los episodios más intensos de tos.

Una vez estabilizado el cuadro se realizó estudio de la función respiratoria del paciente que fue normal y se repitió la TAC persistiendo la impresión diagnóstica de neumonía lipoidea y apreciando una leve mejoría en las imágenes radiológicas.

El paciente fue dado de alta y citado para revisiones posteriores en la consulta de neumología de nuestro hospital; en la actualidad, tras un año de seguimiento, el paciente permanece asintomático y con una vida laboral normal; en la TAC se observan leves secuelas del infiltrado lipoideo sin repercusión funcional (Fig. 2).

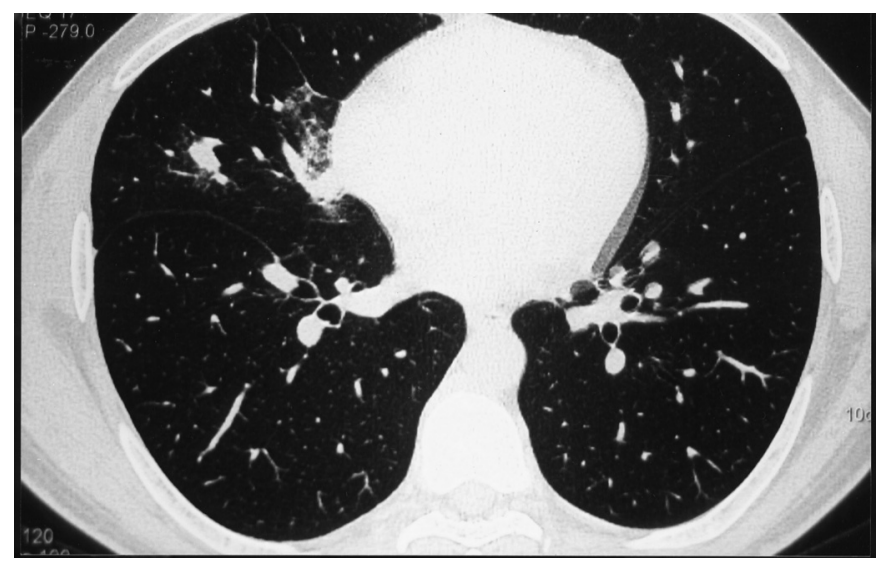

Fig. 2. TAC tras un año de evolución.

\section{DISCUSIÓN}

La neumonía lipoidea es una entidad poco frecuente que se produce como consecuencia del depósito de materiales grasos en el tejido pulmonar; este depósito puede ser de origen exógeno o endógeno.

La forma endógena aparece, sobre todo, como consecuencia de procesos neoplásicos o supurativos crónicos y constituye la forma menos frecuente de neumonía lipoidea; se han documentado casos asociados a enfermedades como la proteinosis alveolar, embolismos grasos, enfermedad mixta del tejido conectivo, secuestro pulmonar o enfermedad de Nieman-Pick (1-3).

Las formas exógenas de la enfermedad pueden producirse por una exposición continuada a materiales oleosos o como consecuencia de una única exposición que desencadena la neumonía a las pocas horas.

La neumonía lipoidea crónica ha sido asociada clásicamente a determinadas prácticas, como en los "comedores de fuego", y a otras exposiciones laborales como pintores y fumigadores; también existen casos asociados al consumo de aceites vegetales o animales en forma de laxantes, pulverizaciones o gotas nasales (4-7)

El caso que se presenta corresponde al desarrollo de una neumonía lipoidea aguda tras la aspiración accidental de una cantidad indeterminada de gasóleo de automóvil en el curso de un maniobra de succión para vaciar el depósito de combustible de un coche sin tomar las debidas precauciones; este tipo de prácticas son bastante frecuentes, y no es raro que en alguna de ellas se llegue a ingerir o, como en este caso, a aspirar una parte del combustible. El gasóleo es un aceite intermedio que se obtiene de la destilación fraccionada del petróleo; esta compuesto por una mezcla de hidrocarburos y se usa, entre otras cosas, como combustible de vehículos.

La neumonía lipoidea aguda suele cursar con fiebre, dolor pleurítico, tos no productiva y condensación radiológica e insuficiencia respiratoria que se establecen a las pocas horas de la aspiración. La presencia del antecedente de exposición es el parámetro fundamental a la hora de establecer el diagnóstico diferencial con las neumonías de origen infeccioso.

Se han descrito casos relacionados con aspiración de diferentes tipos de grasas o materiales oleosos habitualmente debidos a aspiración accidental o disminución del nivel de conciencia; particularmente interesante por sus implicaciones 
prácticas es el caso de una paciente que desarrolló una neumonía aguda a consecuencia de un sondaje nasogástrico utilizando vaselina como lubricante (8-10).

En estos casos, la correcta identificación del factor epidemiológico de riesgo permite un diagnóstico precoz e incluso evita, en algunos casos, la realización de biopsias pulmonares, puesto que la realización de una TAC de alta resolución u otros medios de imagen permite establecer un diagnóstico de sospecha que, asociado al antecedente de exposición, ofrece una buena garantía diagnóstica $(11,12)$.

A pesar de todo ello, el diagnóstico de certeza requiere la demostración histológica, ya sea a partir del material obtenido en el LBA, esputo, punción-aspiración con aguja fina o biopsia pulmonar transbronquial (9).

El paciente de nuestro caso acudió al servicio de urgencias inmediatamente tras la aspiración, presentando únicamente

\section{Bibliografía}

1. Ikehara K, Suzuki M, Tsurabi T, Ishigatsubo Y. Lipoid pneumonia. Lancet 2002; 359: 1300.

2. Nicholson Andrew G, Wells Athol U, Hooper J, Hansell David M, Kelleher Andrea, Morgan C. Successful treatment of endogenous lipoid pneumonia due to Niemann-Pick Type B disease with whole-lung lavage. Am J Respir Crit Care Med 2002; 165: 128-31.

3. Barta Z, Szabo GG, Bruckner G, Szegedi G. Endogenous lipoid pneumonia associated with undifferentiated connective tissue disease (UCTD). Med Sci Monit 2001; 7: 134-6.

4. Brander PE, Taskinen E, Stenius-Aarniala B. Fire-eater's lung. Eur Respir J 1992; 5: 112-4.

5. Gatusso P, Reddy VB, Castelli MJ. Exogenous lipoid pneumonitis due to Vicks Vaporub inhalation diagnosed by fine needle aspiration cytology. Citopathology 1991; 2: 315-6.

6. Berg B, Saenger J. Exogenous lipoid pneumonia. New Engl J Med 1998; 19: 512.

7. Abad A, de Miguel J, López R, Gómez D, Najera L, Jara B. Neumonía lipoidea en relación con exposición laboral a pinturas. Arch Bronconeu- tos seca de características irritativas y una ligera alcalosis respiratoria, sin embargo, la aparición en apenas seis horas de dolor pleurítico y condensación parenquimatosa en la radiografía de tórax permitieron el diagnóstico de neumonía lipoidea apoyado por el antecedente de la aspiración de gasóleo y la TAC de alta resolución.

Esta forma de presentación es menos frecuente que la neumonía lipoidea crónica, que debe ser incluida en el diagnóstico diferencial de las neumonías, sobre todo en las que evolucionan de forma atípica; para ello, es fundamental la realización de una adecuada historia de exposiciones que puede ser la clave de un diagnóstico correcto. Además, debe realizarse un seguimiento de la evolución del paciente ante el posible desarrollo de complicaciones como insuficiencia respiratoria crónica, infecciones por micobacterias, hipercalcemia e incluso cáncer de pulmón $(13,14)$. mol 2003; 39: 133-5.

8. Porcar C, Clemente C, Rubio M. Neumonía lipoidea aguda: Presentación de un nuevo caso. Arch Bronconeumol 1997; 33: 426.

9. Bernabeu R, Méndez P, Abellán MC, Polo LA, Lorenzo M, Sánchez F. Neumonía lipoidea aguda debida a la aspiración accidental de vaselina utilizada en un sondaje nasogástrico. Arch Bronconeumol 2000; 36: 485-7.

10. Lores L, Ribas J, Ruiz J, Cuxart A, Frigola A, Morera J. Neumonía lipoidea aguda. Presentación de dos casos. Med Clin 1996; 106: 747-8.

11. Laurent F, Philippe JC, Vergier B, Granger B, Darpeix B, Vergeret J, et al. Exogenous lipoid pneumonia: HRCT, MR, and pathologic findings. Eur Radiol 1999; 9: 1190-6.

12. Lee JS, Im JG, Song KS, Seo JB, Lim TH. Exogenous lipoid pneumonia: High resolution CT findings. Eur Radiol 1999; 9: 287-91.

13. Felson B, Ralaisomay G. Carcinoma of the lung complicating lipoid pneumonia. AJR 1983; 141: 901-7.

14. Jovannic I, Desves B, Lena H, Quinquenel ML, Donnio PY, Delaval P Exogenous lipoid pneumonia complicated by mycobacterium fortuitum and aspergillus fumigatus infections. Eur Respir J 1996; 9: 172-4. 